\title{
Chemical, Sensory, and Consumer Evaluations of 'DaisySL' Mandarins Grafted onto Three Different Rootstocks
}

Tyler J. Simons ${ }^{1}$, Christopher J. McNeil, Aubrey D. Pham, and Carolyn M. Slupsky

Department of Food Science \& Technology, University of California, Davis, One Shields Avenue, Davis, CA 95616

Mikeal L. Roose

Department of Botany \& Plant Sciences, University of California, Riverside, 900 University Avenue, Riverside, CA 92521

\section{Jean-Xavier Guinard \\ Department of Food Science \& Technology, University of California, Davis, One Shields Avenue, Davis, CA 95616}

Additional index words. Citrus, consumer testing, descriptive analysis, California, adults, children, flavor, nuclear magnetic resonance

\begin{abstract}
DaisySL' mandarins (Citrus reticulata Blanco) grafted to Schaub Rough lemon (C. jambhiri Lush; SHRL) Carrizo citrange (Citrus sinensis Osb. $\times$ Poncirus trifoliata $\mathrm{L}$. Raf.; CARR), and Rubidoux Trifoliate [Poncirus trifoliata (L.) Raf.; RUBT] rootstocks were measured to determine their liking by adults and children, sensory properties, and targeted metabolomics over the course of 2 consecutive years. Chemical measurements showed differences in sugars, acids, and ethanol content, whereas a descriptive analysis found variations in sweetness, mandarin flavor, juiciness, and peelability. During both years, adults significantly preferred 'DaisySL' mandarins grafted to CARR and RUBT over those grafted to SHRL $(P \leq 0.05)$. Children liked the fruit grafted to CARR and RUBT rootstocks significantly more than fruit grafted to SHRL during the first year, but they did not prefer fruit grafted to any rootstock during the second year. This research found that 'DaisySL' mandarins are a well-liked variety of mid-to-late season mandarin capable of filling the seasonal gap between clementine and $W$. Murcott varieties. We concluded that the rootstock can affect the chemical composition, sensory profile, and consumer preferences for 'DaisySL' mandarins.
\end{abstract}

Choosing a rootstock for citrus fruit is a crucial decision made by growers. Important traits that should be considered include tolerance to diseases and pests, scion compatibility, soil and climate conditions, suckering, and precocity, because all of these can affect the yield, quality, and health of the tree (Roose, 2014). A poor choice of rootstock will have negative effects on the plant, including increased disease rates and lower fruit production. Specific rootstocks are known to differ in regard to fruit quality (Roose, 2014), and the choice of rootstock involves chemical, flavor, and liking effects (Benjamin et al., 2013).

The 'DaisySL' mandarin is known for its low seed count, mid-to-late season ripening, medium-to-large fruit size, and excellent flavor (Roose and Williams, 2010). Originating from researchers at the University of

Received for publication 6 Mar. 2019. Accepted for publication 11 Apr. 2019.

This research was funded by the California Citrus Research Board (Project \# 5200-150).

${ }^{1}$ Corresponding author. E-mail: tjsimons@ucdavis.edu.
California, Riverside, the fruit was created from irradiated budwood of the 'Daisy' mandarin (Fortune $\times$ Fremont) and officially released in June 2009. Although it was cited as being of high quality, past assumptions were based on chemical measurements, including ${ }^{\circ}$ Brix and titratable acidity (Roose and Williams, 2010).

Recent research of consumer preferences for mandarins and oranges has shown that preference is dependent on many sensory modalities, such as taste, texture, and flavor attributes (Goldenberg et al., 2015; Obenland et al., 2009; Simons et al., 2018a, 2018 b; Tietel et al., 2011). Few studies have evaluated children's preferences regarding mandarins; however, children are among the target consumers for mandarins (Simons et al., 2018a).

During this study, 'DaisySL' mandarins $(C$. reticulata Blanco) were grown at the University of California Lindcove Research and Extension Center in Exeter, CA. Budwood was grafted to three different common rootstocks found in California: Rough lemon (C. macrophylla, $C$. jambhiri Lush.), Trifoliate [P. trifoliata (L.) Raf.], and Carrizo (C. sinensis L., Osbeck
Poncirus trifoliata L.). In California, oranges from Rough lemon rootstocks have higher yields, but the fruit tend to have lower levels of soluble solids and acid (Roose, 2014). Carrizo and Trifoliate are both considered good to excellent rootstocks for internal fruit quality (sugar, acid, and juice content) in California (Roose, 2014). These rootstocks were chosen for the 'DaisySL' variety due to their known differences in fruit production characteristics, especially fruit quality, for oranges. The aims of this research were to determine the differences in chemical profiles between rootstocks and to determine if these differences caused sensory or consumer liking differences. Because past research revealed higher soluble solids and acids in oranges (Roose, 2014), we hypothesized that the fruit grown on the Carrizo and Trifoliate rootstocks would be preferred by both adults and children.

\section{Materials and Methods}

The research was performed over two seasons, Jan. 2017 and Jan. 2018, using the same experimental design and outcome variables for each season.

Fruit samples. Mandarin fruit from mature 'DaisySL' grafted onto either Schaub Rough lemon (C. jambhiri Lush; SHRL), Carrizo citrange (Citrus sinensis Osb. $\times$ Poncirus trifoliata L. Raf.; CARR), or Rubidoux Trifoliate [Poncirus trifoliata (L.) Raf.; RUBT] were harvested, washed, and dried before packing at the University of California Lindcove Research and Extension Center in Exeter, CA, during Jan. 2017 and Jan. 2018. All tested trees were planted in Sept. 2012 in the same field block. Trees tested during the experiments were not identical during the 2 years. The fruit was shipped to Davis, where it was immediately stored at $5{ }^{\circ} \mathrm{C}$ and $85 \%$ humidity before testing. During the first year, the mandarins underwent an additional waxing step with Fruit-O-Peel wax (Fruit Growers Supply, Valencia, CA) before packing.

During the first year of testing, fruit were harvested and shipped on 11 Jan. 2017, stored at UC Davis on 12 Jan. 2017, and tested between 17 Jan. 2017 and 21 Jan. 2017. During the second year, fruit were harvested and shipped on 4 Jan. 2018, stored at UC Davis on 5 Jan. 2018, and tested between 8 Jan. 2018 and 12 Jan. 2018.

Chemical analysis. During both years of the study, 10 mandarins from each group were selected for chemical analysis via proton nuclear magnetic resonance ( ${ }^{1} \mathrm{H}$ NMR) spectroscopy. The fruit were juiced using OXO Softworks citrus squeezers (OXO, New York, New York), and an aliquot of juice from each fruit was stored at $-80{ }^{\circ} \mathrm{C}$ until filtration and analysis. Before analysis, the juice was thawed at room temperature and centrifuged. The supernatant was then filtered using a prewashed Amicon Ultra- 0.5 Centrifugal Filter Unit with a $3 \mathrm{KD}$ cut-off (Millipore Sigma, Burlington, MA). Then, $207 \mu \mathrm{L}$ of the filtrate was mixed with $23 \mu \mathrm{L}$ of an internal standard consisting of $5 \mathrm{~mm} 3$ - 


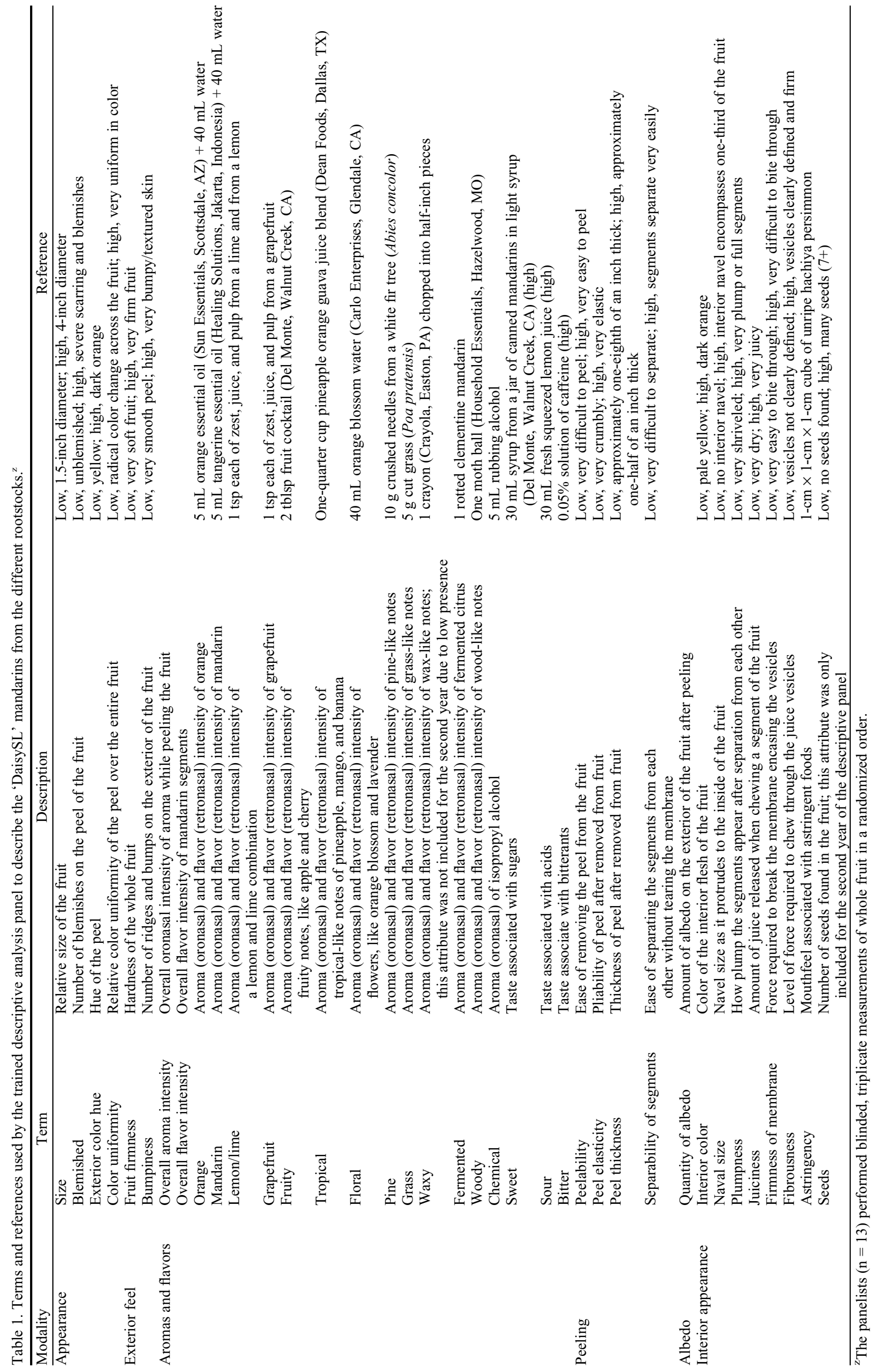


Table 2. Check-all-that-apply (CATA) terms given to the consumers to describe each mandarin.

\begin{tabular}{llll}
\hline \multicolumn{2}{c}{ Adult CATA terms } & \multicolumn{2}{c}{ Child CATA terms } \\
\hline Good appearance & Balanced flavor & Looks good & Watery \\
Bad appearance & Flavorful & Looks bad & Juicy \\
Difficult to peel & Bland & Hard to peel & Dry \\
Easy to peel & Watery & Easy to peel & Mushy \\
Lots of pith & Juicy & Sweet & Bland \\
Aromatic & Mushy & Sour & Chewy \\
Sweet tasting & Chewy & Bitter & \\
Sour tasting & Fibrous & Tasty & \\
Complex flavor & & Flavorful & \\
\hline
\end{tabular}

(trimethylsilyl)-1-propanesulfonic acid-d6 (DSS-d6) dissolved in $>98 \% \mathrm{D}_{2} \mathrm{O}$, to which $0.2 \% \mathrm{NaN}_{3}$ was added to prevent microbial growth (Chenomx, Edmonton, Alberta, Canada). The sample $\mathrm{pH}$ was adjusted to $6.8 \pm$ 0.1 , and $180 \mu \mathrm{L}$ of the sample was transferred to a 3-mm NMR tube. Samples were run on a Bruker Avance 600-MHz NMR spectrometer (Bruker, Billerica, MA) at $298 \mathrm{~K}$ using the Bruker noesypr1d (Chin et al., 2014). The NMR spectra were processed and chemically profiled using Chenomx NMR suite version 8.3. A total of 35 metabolites were profiled for each sample.

Descriptive analysis. A generic descriptive analysis combining elements of a quantitative descriptive analysis and spectrum methods was used and involved 13 judges ( 9 females, 4 males; age range, $25-75$ years) during the first year. During the second year, 11 of the panelists returned and an additional four panelists were recruited. The secondyear descriptive panel consisted of seven females and eight males (age range, 24-75 years).

During the first year, panelists completed nine training sessions for commercial mandarins found in local grocery stores. The first two sessions involved term generation based on commercially available mandarins. The following sessions focused on concept alignment, aided by the use of references, to create a list of descriptive terms (Table 1). During training, judges used a sample ballot that listed each of the terms with an adjacent $10-\mathrm{cm}$ line scale anchored at $1-\mathrm{cm}$ indentations to limit the scale end-use effects. During the second year, the new tasters completed at least five training sessions with the retained panelists; these sessions acted as a refresher. During both years, the performance of the panel (i.e., ability to discriminate, reproducibility, and concept alignment) was checked before performing an evaluation of the fruit using descriptive analysis and a practice evaluation session with fruit from a local grocery store.

Data collection by the panelists was performed using an electronic ballot (FIZZ; Biosystèmes, Couternon, France) for the descriptive analysis during the first year. RedJade software (Tragon Corporation, Redwood Shores CA) was used during the second year. During the evaluation sessions, judges were presented with each whole mandarin and asked to first evaluate the appearance attributes. Then, they were instructed to peel the fruit and evaluate the aroma and peel characteristics by smelling the fruit as they peeled it. Finally, the panelists evaluated the taste, flavor, and texture of the fruit. Unsalted crackers (Mondelez, East Hanover, NJ) and water were provided to cleanse their palates between samples. The panelists were also given a bowl of warm water to clean their hands between samples to prevent carry-over aroma from the peel of the previous sample. The samples were evaluated in triplicate under white light in a three sample $\times$ three replicate design during a single session. The presentation order of the samples was randomized using a William's Latin square design provided by the FIZZ (Biosystèmes) and RedJade (Tragon Corporation) systems.

Consumer tests. Adults and children (ages 7-12 years) from the local communities (Davis, Woodland, and Sacramento, CA) were recruited for the tests. Potential participants were screened for age, allergies, and consumption of citrus. The age of the children was determined based on cognitive abilities required to use hedonic scales, intensity scales, and other sensory measures. Children were accompanied by a parent or guardian at all times, but they were seated in their own individual booths.

The tastings were performed in the Silverado Vineyards Sensory Theater of the Robert Mondavi Institute for Wine and Food Science at the University of California, Davis. Each consumer was given a double-sided paper ballot to evaluate each sample, plain crackers, water, and napkins. Consumers were also given a tray containing six whole mandarins (two from each rootstock). Crackers and water were encouraged as palate cleansers between samples, and napkins were provided for consumers to wipe their hands between samples. Each consumer was asked to first evaluate the appearance, and then to hold the fruit near the nose and peel it to evaluate the aroma before moving tasting it. The samples were blinded with three-digit codes, and the order was randomized using William's Latin square. The use of human subjects was approved by the Institutional Review Board of the University of California, Davis (ID 821014-1).

Adult consumers rated their degree of liking on the 9-point hedonic scale for appearance, overall liking, flavor, and texture, as well as the adequacy of sweetness, sourness, firmness, and juiciness on a 5-point Just About Right (JAR) scale. Child consumers rated their overall liking and liking of the appearance, taste, and texture of the fruit on a smaller 7-point hedonic scale that used faces (Guinard, 2000), as well as the adequacy of the sweetness, sourness, firmness, and juiciness of the fruit on a 3-point JAR scale. Check All That Apply (CATA) attributes (Table 2) were also presented to the consumers with a combination of hedonic and descriptive attributes to use for describing the fruit they tasted.

Data analysis. Data analysis was performed using R (R core team, Vienna, Austria). A full-model, three-factor analysis of variance (ANOVA) was performed for each descriptive attribute. The three factors assessed were judges (J), replications (R), and products $(\mathrm{P})$, along with their two-way interactions $(\mathrm{J} \times \mathrm{R}, \mathrm{J} \times \mathrm{P}$, and $\mathrm{R} \times \mathrm{P})$. When a significant $\mathrm{J} \times \mathrm{P}$ or $\mathrm{P} \times \mathrm{R}$ interaction was found, a pseudo-mixed model was used to assess product differences. Fisher's least significant difference test was performed after the ANOVA to compare product means. For the chemical data, a two-way ANOVA involving the replicate and product was performed, followed by Fisher's least significant difference test.

To evaluate the consumer data, univariate statistics were performed for the hedonic questions. Fisher's least significant difference test was used to determine differences between products. JAR data was compared using the chi-squared test. CATA data was analyzed with Cochran's Q test. Any missing values were replaced with the rounded average of the product for the specific question.

\section{Results}

Of 37 attributes on the scorecard, the trained panelists consistently found nine attributes that differed significantly among the rootstocks during both years of testing: size, mandarin aroma, peelability, peel thickness, interior color, sweetness, overall flavor, mandarin flavor, and juiciness (Table 3). During both years, fruit from CARR and RUBT rootstocks were juicier than fruit from SHRL rootstocks. Fruit from the RUBT rootstock had higher sweetness ratings than fruit from the SHRL rootstock during both years. RUBT had the highest overall flavor rating, highest sweetness rating, the most mandarin flavor, and was the juiciest across both years. Fruit from SHRL had lower ratings for sweetness, mandarin flavor, and juiciness; however, it had high ratings for peel thickness and peelability. The attributes of size, mandarin aroma, and interior color were significantly different during both years, but not consistently across rootstocks.

Only six of the measured compounds differed significantly during both years (Table 4): alanine, citrate, ethanol, galactose, malate, and sucrose. Although not significantly different from the fruit from CARR, fruit from SHRL had the lowest levels of citrate; however, fruit from RUBT had the highest levels of citrate during both years. Fruit from SHRL also had the lowest sucrose values during both years. The average levels 
of fructose and glucose for all the fruit from the first year were not significantly different at $108,870.9 \mu \mathrm{M}$ and $113,868.8 \mu \mathrm{M}$, respectively. During the second year, fruit from CARR and RUBT showed significantly $(P \leq$ $0.05)$ higher fructose and glucose values than fruit from SHRL. Fruit from CARR and RUBT had an average fructose value of $98,769.0 \mu \mathrm{M}$ and an average glucose value of $99,158.8 \mu \mathrm{M}$ compared with fruit from SHRL (fructose, 69,219.1 $\mu \mathrm{M}$; glucose, $66,281.6 \mu \mathrm{M})$. Fruit sampled during the first year had higher concentrations of ethanol, but fruit from CARR and RUBT had higher ethanol values than SHRL. Fruit from CARR and RUBT showed higher alanine values than fruit from SHRL, but this was not true for fruit from RUBT during the second year; however, the difference was not significant. Fruit from SHRL had higher galactose values than fruit from CARR during both years; it also had higher galactose values than fruit from RUBT during the second year. Fruit from SHRL had higher malate values than fruit from CARR or RUBT during both years.

During the first season, 31 children and 70 adults evaluated the mandarins. During the second season, 70 children and 126 adults participated. With the exception of children during the second year, both sets of consumers showed significantly higher liking ratings $(P \leq 0.05)$ for fruit from both the CARR and RUBT rootstocks compared to fruit from the SHRL rootstock (Figs. 1 and 2). Although the children's average scores for fruit from the SHRL rootstock were lower than the average ratings for fruit from either of the other rootstocks, the difference was not statistically significant. Adults did not prefer mandarins from the CARR rootstocks over the RUBT rootstocks during the first year, but they did during the second year.

More significant differences in JAR ratings were determined by adults (Table 5) than by children (data not shown). During the first year, the fruit from the CARR and RUBT rootstocks were rated as just about right for sweetness by $60 \%$ and $64 \%$ of consumers, respectively, compared with a rating of only $46 \%$ for fruit from the SHRL rootstock. Adults also rated the fruit from CARR as having more ideal sourness $(60 \% \mathrm{JAR})$ than fruit from RUBT or SHRL (49\% JAR). During the second year, a similar trend was observed, but fruit from the CARR rootstock had a higher proportion of "just about right" ratings than fruit from the RUBT rootstock. Fruit from the CARR rootstock scored well regarding juiciness during both years. There was no significant difference in the JAR ratings provided by children during either year, with the exception of firmness (data not shown). In agreement with the ratings provided by adults, children rated fruit from the SHRL rootstock as firmer than the other samples during the second year.

Adults and children completed a CATA questionnaire that was printed on the ballot. This CATA section included fruit attributes that they could uses to appropriately describe the sample. The available attributes that the
Table 3. Descriptive attributes and their average ratings for 'DaisySL' mandarins that showed significant differences $\left(P \leq 0.05\right.$ by ANOVA) during both years of testing. ${ }^{z}$

\begin{tabular}{|c|c|c|c|c|c|c|}
\hline \multirow[b]{2}{*}{ Attribute } & \multicolumn{3}{|c|}{ Yr 1} & \multicolumn{3}{|c|}{ Yr 2} \\
\hline & CARR & RUBT & $\overline{\text { SHRL }}$ & $\overline{\text { CARR }}$ & RUBT & $\overline{\text { SHRL }}$ \\
\hline$\overline{\text { Size }}$ & $6.29 \mathrm{~b}$ & $5.14 \mathrm{c}$ & $7.84 \mathrm{a}$ & $5.22 \mathrm{a}$ & $3.83 \mathrm{~b}$ & $4.69 \mathrm{ab}$ \\
\hline Mandarin aroma & $4.48 \mathrm{~b}$ & $4.79 \mathrm{~b}$ & $5.45 \mathrm{a}$ & $4.40 \mathrm{~b}$ & $4.98 \mathrm{a}$ & $4.26 \mathrm{~b}$ \\
\hline Peelability & $2.23 \mathrm{~b}$ & $2.69 \mathrm{~b}$ & $3.92 \mathrm{a}$ & $4.04 \mathrm{a}$ & $2.67 \mathrm{~b}$ & $4.50 \mathrm{a}$ \\
\hline Peel thickness & $2.89 \mathrm{~b}$ & $2.98 \mathrm{~b}$ & $5.73 \mathrm{a}$ & $3.94 \mathrm{~b}$ & $2.54 \mathrm{c}$ & $5.26 \mathrm{a}$ \\
\hline Interior color & $6.29 \mathrm{a}$ & $6.27 \mathrm{a}$ & $5.19 \mathrm{~b}$ & $4.02 \mathrm{~b}$ & $4.44 \mathrm{a}$ & $3.98 \mathrm{~b}$ \\
\hline Sweetness & $7.43 \mathrm{ab}$ & $8.06 \mathrm{a}$ & $6.88 \mathrm{~b}$ & $6.80 \mathrm{a}$ & $6.85 \mathrm{a}$ & $6.27 \mathrm{~b}$ \\
\hline Overall flavor & $6.73 \mathrm{~b}$ & $7.38 \mathrm{a}$ & $5.40 \mathrm{c}$ & $6.13 b$ & $6.89 \mathrm{a}$ & $6.24 b$ \\
\hline Mandarin flavor & $4.65 \mathrm{~b}$ & $5.48 \mathrm{a}$ & $4.08 \mathrm{~b}$ & $6.10 \mathrm{~b}$ & $6.66 \mathrm{a}$ & $5.59 \mathrm{c}$ \\
\hline Juiciness & $6.19 \mathrm{a}$ & $6.55 \mathrm{a}$ & $4.74 \mathrm{~b}$ & $6.45 \mathrm{a}$ & $6.67 \mathrm{a}$ & $5.76 \mathrm{~b}$ \\
\hline
\end{tabular}

${ }^{\mathrm{z}}$ The panelists rated the attributes on a 10 -cm line scale $(\mathrm{low}=1$ and high $=10)$. Attributes in the same row and year sharing a letter are not significantly $(P>0.05)$ different according to Fisher's least significant difference test.

Table 4. Chemical compounds that showed significant differences in the three rootstocks during both years of testing. ${ }^{\mathrm{z}}$

\begin{tabular}{lrrrrrrr}
\hline & \multicolumn{4}{c}{ Yr 1 } & & \multicolumn{3}{c}{ Yr 2 } \\
\cline { 2 - 4 } \cline { 7 - 8 } Compound & \multicolumn{1}{c}{ CARR } & \multicolumn{1}{c}{ RUBT } & \multicolumn{1}{c}{ SHRL } & & CARR & \multicolumn{1}{c}{ RUBT } & \multicolumn{1}{c}{ SHRL } \\
\hline Alanine & $576.33 \mathrm{a}$ & $553.85 \mathrm{a}$ & $428.44 \mathrm{~b}$ & & $802.73 \mathrm{a}$ & $737.57 \mathrm{ab}$ & $592.57 \mathrm{~b}$ \\
Citrate & $34,631.54 \mathrm{~b}$ & $44,811.06 \mathrm{a}$ & $28,941.79 \mathrm{~b}$ & & $35,794.58 \mathrm{~b}$ & $46,296.94 \mathrm{a}$ & $30,087.68 \mathrm{~b}$ \\
Ethanol & $9,160.64 \mathrm{a}$ & $7,931.99 \mathrm{a}$ & $5,010.35 \mathrm{~b}$ & $2,218.44 \mathrm{a}$ & $2,031.83 \mathrm{a}$ & $586.51 \mathrm{~b}$ \\
Galactose & $297.46 \mathrm{ab}$ & $221.59 \mathrm{~b}$ & $368.89 \mathrm{a}$ & $114.00 \mathrm{~b}$ & $133.30 \mathrm{~b}$ & $193.16 \mathrm{a}$ \\
Malate & $9,133.46 \mathrm{~b}$ & $8,487.36 \mathrm{~b}$ & $12,224.03 \mathrm{a}$ & $9,248.72 \mathrm{~b}$ & $9,778.40 \mathrm{~b}$ & $14,549.42 \mathrm{a}$ \\
Sucrose & $198,833.05 \mathrm{a}$ & $202,110.90 \mathrm{a}$ & $184,237.51 \mathrm{~b}$ & & $160,814.14 \mathrm{a}$ & $173,765.04 \mathrm{a}$ & $131,074.86 \mathrm{~b}$ \\
\hline
\end{tabular}

${ }^{\mathrm{z}}$ All concentrations are shown in $\mu \mathrm{M}$. Values sharing a letter for each year in each row are not significantly different $(P>0.05)$ according to Fisher's least significant difference test.
A

9

8

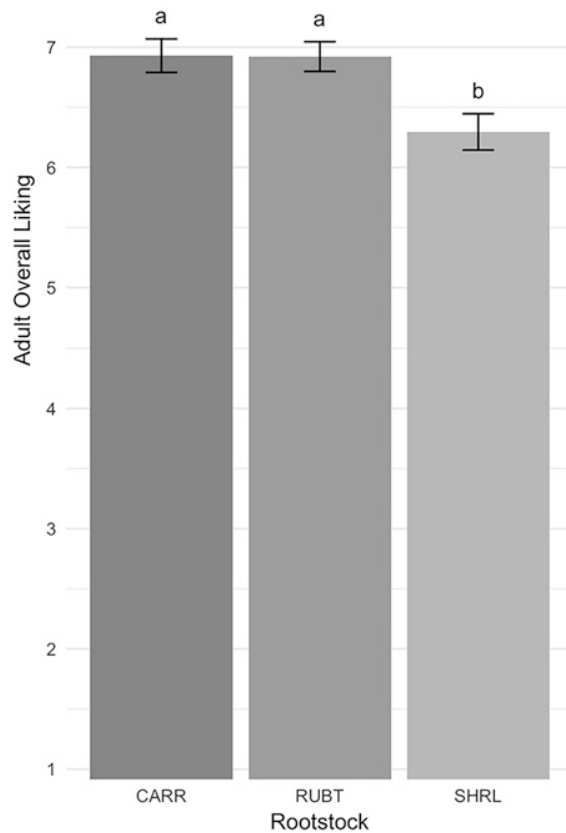

B
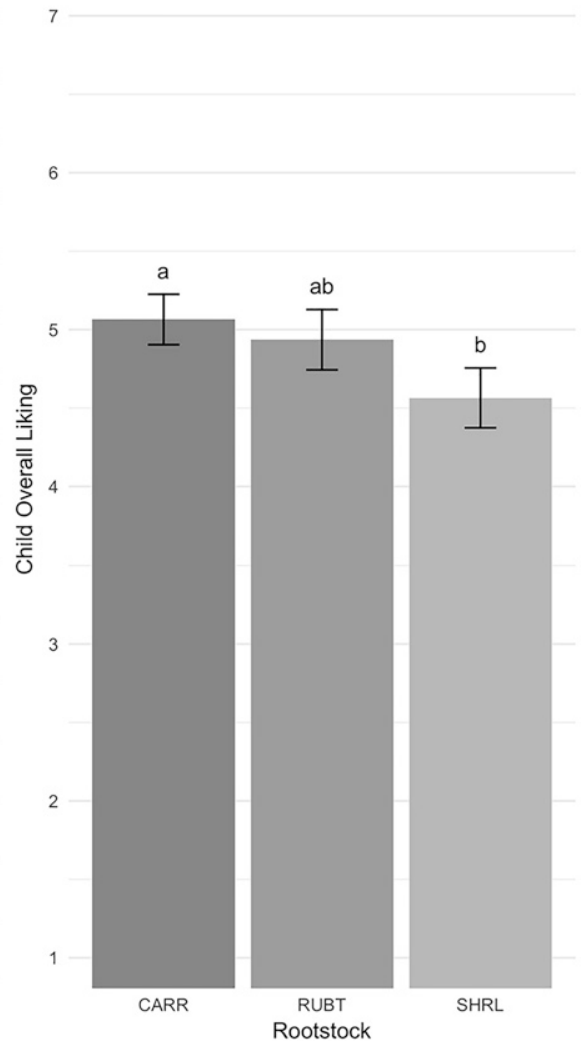

Fig. 1. (A) Overall liking by the adult consumers $(n=70)$ of the 'DaisySL' mandarins during the first year of testing. Bars with a different letter are significant $(P \leq 0.05)$ according to Fisher's least significant difference test. (B) Overall liking by the child consumers $(n=31)$ of the 'DaisySL' mandarins during the first year of testing. Bars with a different letter are significant $(P \leq 0.05)$ according to Fisher's least significant difference test. 
A

9

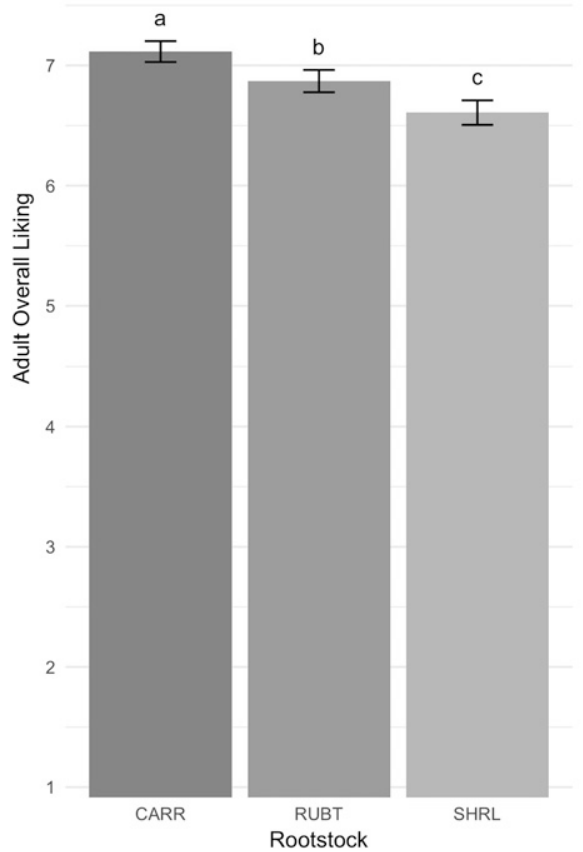

6

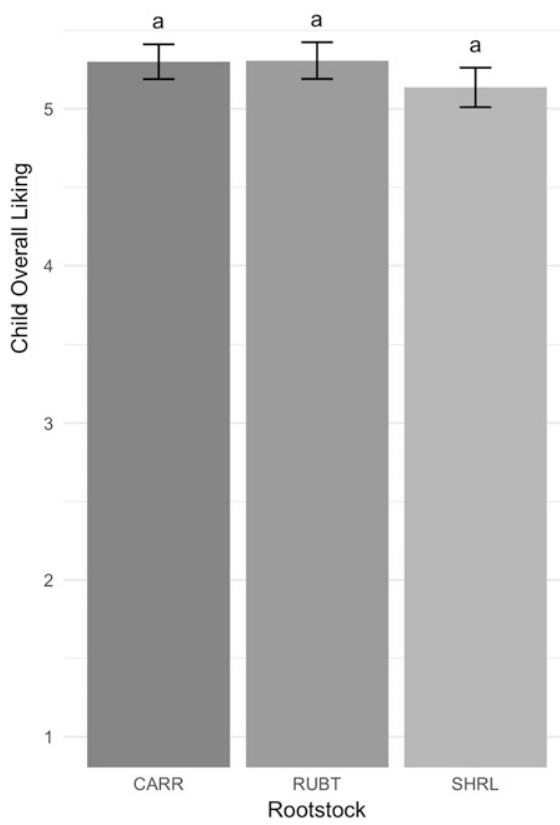

Fig. 2. (A) Overall liking by the adult consumers $(\mathrm{n}=126)$ of the 'DaisySL' mandarins during the second year of testing. Bars with a different letter are significant $(P \leq 0.05)$ according to Fisher's least significant difference test. (B) Overall liking by the child consumers $(\mathrm{n}=70)$ of the 'DaisySL' mandarins during the second year of testing. Bars with a different letter are significant $(P \leq 0.05)$ according to Fisher's least significant difference test.

Table 5. Adult Just-About-Right (JAR) attribute ratings. ${ }^{\mathrm{z}}$

\begin{tabular}{|c|c|c|c|c|c|c|c|}
\hline \multirow[b]{2}{*}{ Attribute } & \multirow[b]{2}{*}{ Intensity } & \multicolumn{3}{|c|}{ Yr $1(n=70)$} & \multicolumn{3}{|c|}{$\operatorname{Yr} 2(n=126)$} \\
\hline & & $\overline{\mathrm{CARR}}$ & RUBT & $\overline{\text { SHRL }}$ & $\overline{\text { CARR }}$ & RUBT & $\overline{\text { SHRL }}$ \\
\hline \multirow{3}{*}{ Sweetness } & Too little & $22 \%$ & $20 \%$ & $38 \%$ & $19 \%$ & $29 \%$ & $43 \%$ \\
\hline & JAR & $65 \%$ & $60 \%$ & $46 \%$ & $72 \%$ & $64 \%$ & $52 \%$ \\
\hline & Too much & $13 \%$ & $20 \%$ & $15 \%$ & $10 \%$ & $7 \%$ & $5 \%$ \\
\hline \multirow{3}{*}{ Sourness } & Too little & $29 \%$ & $36 \%$ & $44 \%$ & $16 \%$ & $20 \%$ & $21 \%$ \\
\hline & JAR & $60 \%$ & $49 \%$ & $49 \%$ & $72 \%$ & $63 \%$ & $54 \%$ \\
\hline & Too much & $11 \%$ & $15 \%$ & $7 \%$ & $12 \%$ & $17 \%$ & $24 \%$ \\
\hline \multirow{3}{*}{ Juiciness } & Too little & $6 \%$ & $10 \%$ & $16 \%$ & $6 \%$ & $4 \%$ & $17 \%$ \\
\hline & JAR & $81 \%$ & $80 \%$ & $70 \%$ & $84 \%$ & $74 \%$ & $78 \%$ \\
\hline & Too much & $13 \%$ & $10 \%$ & $13 \%$ & $10 \%$ & $22 \%$ & $5 \%$ \\
\hline \multirow{3}{*}{ Firmness } & Too little & $18 \%$ & $14 \%$ & $23 \%$ & $21 \%$ & $31 \%$ & $13 \%$ \\
\hline & JAR & $77 \%$ & $79 \%$ & $67 \%$ & $73 \%$ & $62 \%$ & $73 \%$ \\
\hline & Too much & $5 \%$ & $7 \%$ & $10 \%$ & $7 \%$ & $7 \%$ & $14 \%$ \\
\hline
\end{tabular}

${ }^{2}$ Percentages represent the proportion of adults who rated the attributes as too low, just right or too high for fruit on each rootstock. Bold values denote a significant difference $(P \leq 0.05)$ according to the chi-squared test between the rootstocks and the JAR proportions for each attribute during that year only. For each attribute, values in columns may not add up to exactly $100 \%$ due to rounding.

Table 6. CATA (check-all-that-apply) attributes rated by adult consumers that showed significant $(P \leq$ 0.05 ) differences across both years of testing by Cochran's $Q$ test.

\begin{tabular}{|c|c|c|c|c|c|c|}
\hline \multirow[b]{2}{*}{ Attribute } & \multicolumn{3}{|c|}{ Yr 1} & \multicolumn{3}{|c|}{ Yr 2} \\
\hline & $\overline{\text { CARR }}$ & RUBT & $\overline{\text { SHRL }}$ & $\overline{\text { CARR }}$ & RUBT & $\overline{\text { SHRL }}$ \\
\hline$\overline{\text { Sour }}$ & $21 \%$ & $18 \%$ & $10 \%$ & $20 \%$ & $28 \%$ & $\overline{32 \%}$ \\
\hline Flavorful & $51 \%$ & $49 \%$ & $36 \%$ & $55 \%$ & $45 \%$ & $45 \%$ \\
\hline Bland & $6 \%$ & $14 \%$ & $33 \%$ & $5 \%$ & $10 \%$ & $13 \%$ \\
\hline Watery & $11 \%$ & $8 \%$ & $20 \%$ & $9 \%$ & $17 \%$ & $10 \%$ \\
\hline Juicy & $69 \%$ & $58 \%$ & $49 \%$ & $73 \%$ & $71 \%$ & $56 \%$ \\
\hline
\end{tabular}

HortSCIEnCE Vol. 54(7) July 2019

children and adults could choose from are shown in Table 2. Adults found many CATA attributes to be significantly different between the samples, but only five were consistent across both years (Table 6). The attributes "flavorful" and "juicy" had the same trend across both years; fruit from the CARR and RUBT rootstocks had higher scores for these two attributes than fruit from the SHRL rootstock. Fruit from the SHRL rootstock received more "bland" ratings than fruit from either of the other rootstocks. The consumermarked CATA attributes of "sour tasting" and "watery" showed differences across the samples during both years, but these differences were not consistent, meaning that the same fruit did not have the highest scores during both years. Regarding the ratings provided by children, only two CATA attributes were found to be significant between rootstocks (data not shown). During the first year, the children considered the fruit from the SHRL rootstock to be less "sour" and "tasty" than the other two samples. During the second year, children reported that fruit from the RUBT and SHRL rootstocks had a better appearance than fruit from the CARR rootstock. However, neither observation was consistent across both years.

\section{Discussion}

The descriptive panelists were trained to evaluate 37 different attributes encompassing a range of sensory modalities, including appearance, taste, flavor, texture, and peelability. Using these metrics, only nine attributes were found to differ between 'DaisySL' mandarins with regard to rootstock. These attributes included some of the most important sensory modalities of mandarins, including sweetness, overall flavor, juiciness, peelability, and mandarin flavor.

The significant sensory attributes were related to many of the chemical markers that were found to be different among rootstocks. Although only 5 of the 35 tested compounds differed significantly among the rootstocks, they are known to be important for mandarin flavor; these included sucrose, ethanol, and citrate, and others (Tietel et al., 2011). Differences in sucrose (and fructose and glucose during the second year) can help explain the differences in sweetness detected by the descriptive panel in their intensity ratings and by consumers in their JAR ratings. Interestingly, despite higher levels of citrate in fruit from the RUBT rootstock (Table 4), this sample was rated significantly sweeter than fruit from SHRL rootstock. Because the fruit were not stored for particularly long periods, the higher juice ethanol content from the first-year fruit may have been due to ripeness caused by differing weather patterns between the 2 years. Despite the ethanol difference, wax and storage did not seem to affect the other compounds tested (Table 4), descriptive ratings (Table 3 ), or consumer liking (Figs. 1 and 2).

Consumer panels and descriptive analyses showed overall positive responses to the fruit 
tested during both years, despite the small differences between the rootstocks. The hedonic ratings reported by adults placed them among the highest rated fruit tested during the 2015-2016 commercial mandarin season (Simons et al., 2018a). The high values of sweetness, overall flavor, mandarin flavor, and juiciness had strong positive effects on consumer preference. All samples, except for fruit from the SHRL rootstock during the first year, had higher overall liking ratings provided by adults than any commercial mandarin tested during Spring 2016 (Simons et al., 2018a). Another reason why these samples were scored so well by the adults was because of their attribute adequacy, as determined by the JAR questions. For almost all samples, the majority of adult consumers found the sweetness, sourness, firmness, and juiciness of the fruit to be just about right. The children did not necessarily agree with the high liking ratings provided by the adults, however. On average, the children gave overall liking ratings of $\leq 5.5$ out of 7 . These ratings were lower than those for one clementine and two W. Murcott samples tasted during a prior study (Simons et al., 2018a).

There are many ways to explain the high liking ratings provided by the adults, including variety, freshness, minimal storage, and good farming practices, all of which can affect the final flavor of the fruit (Goldenberg et al., 2015; Tietel et al., 2011). However, the high sugar and acid values of the fruit undoubtedly had a large role in the strong consumer acceptance. Measurements of both the chemical and sensory attributes of the fruit are vital for proving chemical differences and determining if these differences are associated with changes in sensory properties. The fruit tested during this study showed significantly different levels of important chemical indicators for flavor, such as sucrose, fructose, and citric acid. These varying levels did affect the sensory properties and consumer preferences. Going forward, it will be critical to measure both chemical and sensory properties together because a significant difference detected in chemical abundance alone does not necessarily indicate a difference in preference.

The children had lower acceptance of the fruit, perhaps because they were used to the flavor and ease of peeling of clementine or W. Murcott mandarins, which are the current commercial standard. The adults, in contrast, may have been more open to the prospect of a new variety. This openness to new varieties by adults and the lukewarm reaction from children might be explained by food neophobia, which decreases with age (Pliner and Salvy, 2006). Food neophobia is common in children and is gener- ally described as the practice of avoiding foods because they are new to them (Dovey et al., 2008). It seems plausible that the children were so used to Cuties and Halos that their association and experience with mandarins mainly involved clementine and W. Murcott varieties. Therefore, adults may be a better group of consumers to market new varieties to in the future.

Past work has evaluated citrus on different rootstock to evaluate important parameters like yield efficiency, sugar content, acid content, rind thickness, juice content, weight, and fruit size (Cantuarias-Avilés et al., 2010; Castle et al., 2010; Cerda et al., 1990; Darjazi, 2009; Fallahi and Rodney, 1992; Mourão Filho et al., 2007; Navarro et al., 2010). However, few studies have evaluated the effects that different rootstocks have on consumer preference. This test confirmed that the 'DaisySL' variety grown on different rootstocks has differing chemical profiles but is a well-liked, mid-to-late season mandarin capable of filling the gap between the popular clementine and W. Murcott seasons (Roose and Williams, 2010). High just about right ratings for sweetness, sourness, firmness, and juiciness across all of the rootstocks showed that this variety performed quite well over a span of 2 years according to many consumers. In addition to high overall liking values and desirable CATA attributes, fruit from the 'DaisySL' performed very well when stored for short periods under nearly ideal conditions. Regarding growth in the future, California growers might want to avoid the SHRL rootstocks if possible. Fruit from these rootstocks showed significantly lower sucrose and lower citrate concentrations, which are both expected outcomes of this particular rootstock (Roose, 2014), and had reduced consumer acceptance. The CARR and RUBT rootstocks both appear to be better choices in terms of consumer liking. However, because the short window between harvest and service used in this study would likely be difficult for growers, packers, and distributors to match, future work should investigate how the 'DaisySL' matches consumer preference when it is commercially shipped, packed, and stored for longer periods of time.

\section{Literature Cited}

Benjamin, G., Z. Tietel, and R. Porat. 2013. Effects of rootstock/scion combinations on the flavor of citrus Fruit. J. Agr. Food Chem. 61:1128611294.

Cantuarias-Avilés, T., F. de A.A. Mourão Filho, E.S. Stuchi, S.R. da Silva, and E. Espinoza-Núñez. 2010. Tree performance and fruit yield and quality of 'Okitsu' Satsuma mandarin grafted on 12 rootstocks. Scientia Hort. 123:318-322.
Castle, W.S., J.C. Baldwin, R.P. Muraro, and R. Littell. 2010. Performance of 'Valencia' sweet orange trees on 12 rootstocks at two locations and an economic interpretation as a basis for rootstock selection. HortScience 45:523-533.

Cerda, A., M. Nieves, and M.G. Guillen. 1990. Salt tolerance of lemon trees as affected by rootstock. Irrig. Sci. 11:245-249.

Chin, E.L., D.O. Mishchuk, A.P. Breksa, and C.M Slupsky. 2014. Metabolite signature of Candidatus Liberibacter asiaticus infection in two citrus varieties. J. Agr. Food Chem. 62:65856591.

Darjazi, B. 2009. The effects of rootstock on the volatile flavour components of page mandarin [(C. Reticulata var dancy $\times$ C. Paradisi var dancan $) \times$ C. Clemantina] juice and peel. Iran J. Chem. Chem. Eng. 28:13.

Dovey, T.M., P.A. Staples, E.L. Gibson, and J.C.G. Halford. 2008. Food neophobia and 'picky/ fussy' eating in children: A review. Appetite 50:181-193.

Fallahi, E. and D.R. Rodney. 1992. Tree size, yield, fruit quality, and leaf mineral nutrient concentration of 'Fairchild' mandarin on six rootstock. J. Amer. Soc. Hort. Sci. 117:28-31.

Goldenberg, L., Y. Yaniv, T. Kaplunov, A. DoronFaigenboim, N. Carmi, and R. Porat. 2015. Diversity in sensory quality and determining factors influencing mandarin flavor liking. J. Food Sci. 80:S418-S425.

Guinard, J.-X. 2000. Sensory and consumer testing with children. Trends Food Sci. Technol. 11:273-283.

Mourão Filho, F. de A.A., E. Espinoza-Núñez, E.S. Stuchi, and E.M.M. Ortega. 2007. Plant growth, yield, and fruit quality of 'Fallglo' and 'Sunburst' mandarins on four rootstocks. Scientia Hort. 114:45-49.

Navarro, J.M., J.G. Pérez-Pérez, P. Romero, and P. Botía. 2010. Analysis of the changes in quality in mandarin fruit, produced by deficit irrigation treatments. Food Chem. 119:1591-1596.

Obenland, D., S. Collin, B. Mackey, J. Sievert, K. Fjeld, and M.L. Arpaia. 2009. Determinants of flavor acceptability during the maturation of navel oranges. Postharvest Biol. Technol. 52:156-163.

Pliner, P. and S. Salvy. 2006. Food neophobia in humans. Psychol. Food Choice CABI Oxfs. 75-92.

Roose, M. 2014. Rootstocks. Citrus production manual. ANR Publications, Oakland, CA.

Roose, M.L. and T.E. Williams. 2010. Mandarin tree named 'DaisySL'. U.S. Patent Application No. 12/456,783.

Simons, T., H. Sivertsen, and J.-X. Guinard. 2018a. Mapping the preferences of adult and child consumers for California-grown mandarins. HortScience 53:1029-1037.

Simons, T., H. Sivertsen, and J.-X. Guinard. 2018b. Mapping the preferences of adult and child consumers for California-grown navel oranges. HortScience 53:661-668.

Tietel, Z., A. Plotto, E. Fallik, E. Lewinsohn, and R. Porat. 2011. Taste and aroma of fresh and stored mandarins. J. Sci. Food Agr. 91:14-23. 